\title{
Acessibilidade em Ambientes Virtuais de Aprendizagem
}

\author{
Carlos Luís ${ }^{1}$, Álvaro Rocha ${ }^{1}$, Maria José Marcelino ${ }^{1}$ \\ cjluis@student.dei.uc.pt, amrocha@dei.uc.pt, zemar@dei.uc.pt \\ ${ }^{1}$ Departamento de Engenharia Informática, FCT, Universidade de Coimbra. Pólo II - Pinhal de Marrocos, \\ 3030-290 Coimbra, Portugal
}

DOI: 10.17013/risti.25.54-65

\begin{abstract}
Resumo: As revoluções tecnológicas e o uso alargado das Tecnologias de Informação e Comunicação têm estimulado a criação de ambientes virtuais de aprendizagem, complementares a todo o processo ensino/aprendizagem. Com este trabalho pretende-se identificar, analisar e descrever alguns métodos existentes para melhorar a acessibilidade dos ambientes virtuais de aprendizagem. Para isso procedeu-se a uma revisão sistemática da literatura sobre acessibilidade nestes ambientes, entre 2010 e 2016, efetuando uma pesquisa em diversas bases de dados de publicações científicas. A combinação de vários métodos com base em padrões e normas é decisiva para apoiar serviços personalizados de ensino/aprendizagem. Por outro lado, o "Universal Design for Learning" е о "Universal Design of Instruction," fornecem um conjunto de princípios e estratégias que procuram reduzir as barreiras e criar modelos facilitadores de ensino/aprendizagem com este propósito. Ainda a integração de práticas pedagógicas inclusivas com recursos tecnológicos adequados permite criar ambientes virtuais de aprendizagem acessíveis.
\end{abstract}

Palavras-Chave: Acessibilidade; ambientes virtuais de aprendizagem; Moodle.

\section{Accessibility in Virtual Learning Environments}

\begin{abstract}
Technological revolutions and the widespread use of Information and Communications Technologies have stimulated the creation of virtual learning environments complementary to the teaching/learning process. The main objective of this paper is to identify, analyseand describe existing methods to improvetheaccessibility of virtual learning environments. To achieve this goal, a systematic review of the literature on accessibility and virtual learning environments was carried out, between 2010 and 2016, using diverse databases of scientific publications. The combination of various methods based on standards and norms is critical to support personalized teaching/learning services. On the other hand, Universal Design for Learning and the Universal Design of Instruction provide a set of principles and strategies that seek to reduce barriers and create facilitating models of teaching / learning for this purpose. Also, the integration of inclusive pedagogical practices with adequate technological resources allows the creation of accessible virtual learning environments.
\end{abstract}

Keywords: Accessibility; virtual learning environments; Moodle. 


\section{Introdução}

As revoluções tecnológicas e o uso alargado das Tecnologias de Informação e Comunicação (TIC) têm estimulado a emergência de novos ambientes virtuais de aprendizagem (AVA), conceito amplo que, para além de integrar todo o tipo de recursos digitais, define a forma de comunicação e os diferentes cenários de educação-formação (Keller, 2005) (Rodrigues et al., 2017). Segundo Dillenbourg (Dillenbourg et al., 2002), as condições necessárias para um AVA são a existência de um espaço concebido para a disponibilidade de informação, a existência de um ambiente com interações sociais e educacionais e de alunos ativos $-\mathrm{e}$ não apenas recetores da informação. A maioria dos AVA permite a utilização de tecnologias diferentes e várias abordagens pedagógicas. Um AVA pode incluir várias funções, tais como: texto, multimédia, chats, fóruns de discussão, perguntas e exames eletrónicos, consultas, emails e bibliotecas com disponibilização de hiperligações (Dillenbourg et al., 2002).

Todo o processo de ensino/aprendizagem vive complexos momentos de mudança. O desenvolvimento das novas tecnologias na educação a distância, torna os AVA um espaço de inovação na construção do conhecimento para todos. Um dos fatores cruciais nos AVA é a sua acessibilidade. Para concretizar esse objetivo e com o intuito de aumentar a sua abrangência é crucial analisar, avaliar e refletir na produção de AVA acessíveis.

A preocupação com a acessibilidade não é um conceito novo (Rocha et al., 2012). Em 1971, na Faculdade de Engenharia da Universidade de Wisconsin-Madison, foi criado o Trace Research \& Development Center para eliminar barreiras e capitalizar as novas oportunidades patenteadas pelas TIC (Center, 2013).

Em 1997, a Australian Human Rights Commission ("the Commission") criou standards de acessibilidade. Ao mesmo tempo, o World Wide Web Consortium ( $\mathrm{W}_{3} \mathrm{C}$ ), fundado por Tim Berners-Lee em 1994, tenta assegurar o crescimento e o desenvolvimento da Web para todos, com o desenvolvimento de protocolos e diretrizes. Estes avanços/progressos permitiram o acesso à informação a todos os utilizadores, independentemente das suas necessidades específicas (W3C, 2005).

Neste artigo apresenta-se uma revisão sistemática da literatura que pretende sintetizar o conhecimento disponível sobre acessibilidade em AVA e, assim, contribuir para uma utilização mais ampla e abrangente destes ambientes.

A metodologia utilizada foi a metodologia PRISMA (Preferred Reporting Items for Systematic Reviwes and Meta-Analyses) (Liberati et al., 2009), que envolve a seleção de artigos versando o tema, de modo a contribuir para um melhor conhecimento sobre o mesmo.

\section{Objetivo e métodos}

O principal objetivo deste artigo é, de uma forma sintética, responder à questão de investigação: Existem métodos para a construção de ambientes virtuais de aprendizagem acessíveis?

Neste contexto, tentou-se identificar, analisar e descrever os métodos utilizados para melhorar a sua acessibilidade, de forma a perspetivar uma das atividades mais antigas da condição dos seres humanos: a aprendizagem. 
O desenho metodológico definido para levar a cabo esta revisão sistemática da literatura seguiu o protocolo PRISMA (Liberati etal., 2009) (Moher, Liberati, Tetzlaff, Altman, \& Grp, 2009), com as suas várias fases: a) definição da questão de investigação; b) identificação dos termos de pesquisa e realização da pesquisa nas bases de dados; c) definição dos critérios de elegibilidade; e d) decisão da inclusão, tendo por base, sequencialmente, o título, os resumos, as conclusões e, numa fase final, a leitura integral dos artigos.

\subsection{Critérios de elegibilidade}

Foram incluídos artigos publicados entre 2010 e 2016, em língua inglesa ou portuguesa, que avaliam e/ou determinam a acessibilidade dos AVA.

\subsection{Fontes de informação}

Foi realizada uma pesquisa nas bases de dados: IEEE Xplore Digital Library (Institute of Electrical and Electronics Engineers, organização fundada em 1884), ACM (Association for Computer Machinery) Digital Library, SCiELO (Scientific Electronic Library Online), ScienceDirect e B-on - Biblioteca do Conhecimento.

Para a pesquisa, foram utilizadas as seguintes palavras-chaves: Acessibilidade and (ambientes virtuais de aprendizagem) e acessibilidade and Moodle (pelo facto de ser um dos AVA's, open source, mais utilizado do mundo), accessibility and (virtual learning environment), Accessibility and Moodle. Foi ainda efetuada uma pesquisa manual nas referências bibliográficas dos artigos consultados.

\subsection{Seleção dos estudos}

Foi feita uma seleção inicial dos artigos com base no título, resumo, conclusão e, posteriormente, leitura dos artigos na íntegra, utilizando os critérios de elegibilidade.

\section{Resultados}

Numa primeira fase, foram identificados 82 artigos, dos quais 49 foram excluídos após avaliação com base no título, resumo e conclusão, uma vez que divergiam do pretendido (Tabela 1).

\begin{tabular}{llll}
\hline Motor de Busca & N.o & Não & Sim \\
\hline ACM Digital Library & 6 & 6 & - \\
\hline IEEE Digital Library & 39 & 23 & 16 \\
\hline SCiELO Scientific Electronic Library Online & 6 & 5 & 1 \\
\hline B-on - Biblioteca do Conhecimento & 15 & 8 & 7 \\
\hline ScienceDirect & 10 & 7 & 3 \\
\hline Outras & 6 & - & 6 \\
\hline Total & 82 & 49 & 33 \\
\hline
\end{tabular}

Tabela 1 - Dados quantitativos relativos às pesquisas efetuadas 
Após leitura na íntegra dos 33 últimos artigos, 24 foram excluídos. Foram, então, selecionados 9 artigos para o estudo proposto (ver Figura 1).

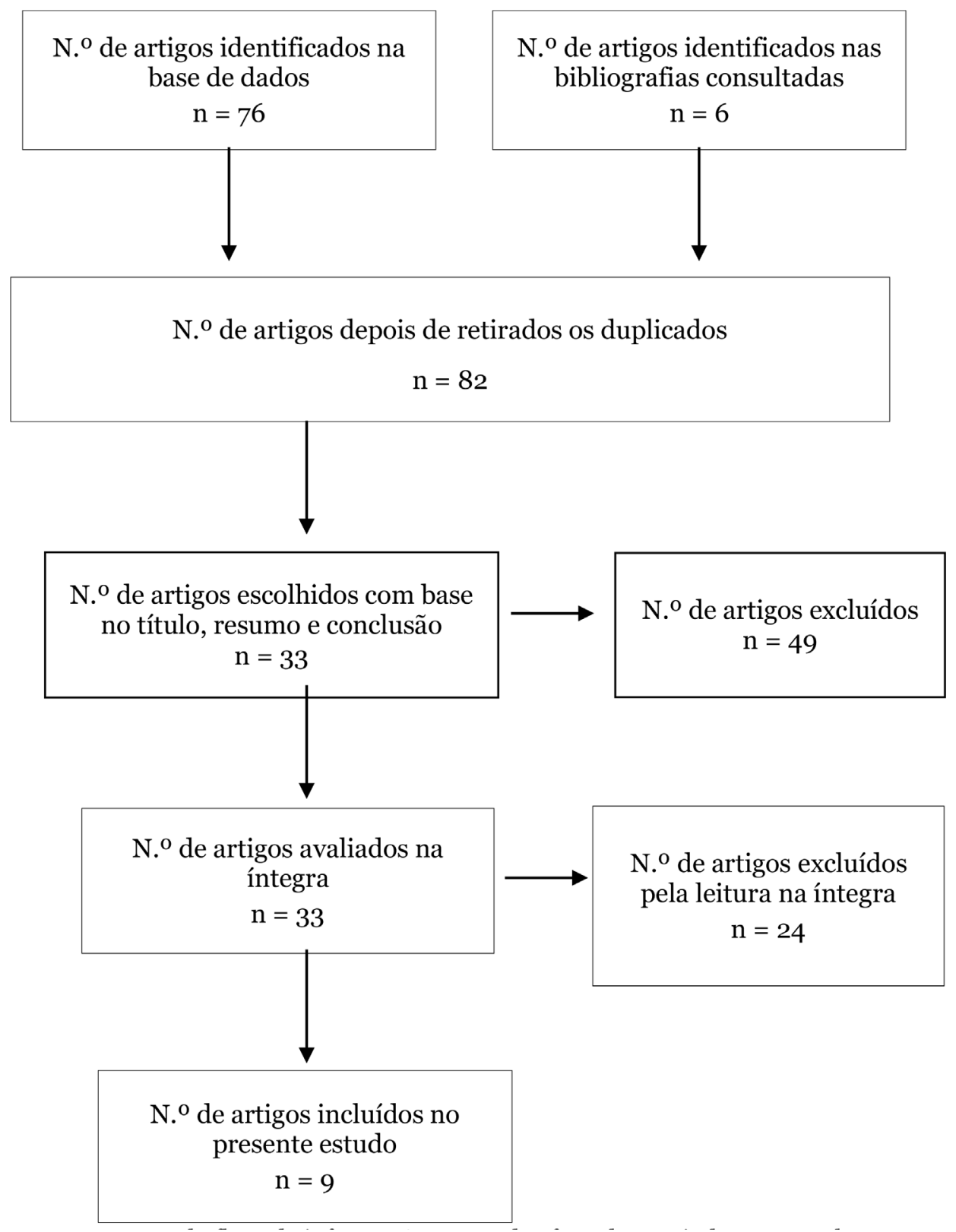

Figura 1 - Esquema do fluxo da informação no estudo efetuado seguindo o protocolo PRISMA(Liberati et al., 2009). 


\section{Autores}

(ano)

H. R. Amado-

Salvatierra, R.

Hernández, and J. R.

Hilera

(2012)

\section{Titulo do artigo}

Implementation of

Accessibility Standards

in the Process of Course

Design in Virtual Learning

Environments
Público-alvo Métodos usados

Estudante sem

Universal Design for Learning

especificação

Universal Design of Instruction
H. R. Amado-

Salvatierra, R.

Hernández, and J. R.

Hilera

(2014)
Teaching and promoting web accessibility in virtual learning environments: A staff training experience in Latin-America
Estudantes do ensino superior
Universal Design for Learning Universal Design of Instruction
H. R. Amado-

Salvatierra and R. H.

Rizzardini

(2014)
Towards a methodology to inclusive curriculum design: An experience presented within an accessible virtual learning environment
Estudantes do ensino superior

Universal Design for Learning

Universal Design of Instruction

P. McAndrew and R.
F. M. Cooper
(2012)
(2012)

Adapting online learning resources for all: planning for professionalism in accessibility

\section{Estudantes do} ensino superior
Universal Design for Learning

$\begin{array}{ll}\text { F. Ravanelli and I. } & \text { Didactic and Pedagogical } \\ \text { Serina } & \text { View of E-learning Activities } \\ \text { (2014) } & \text { Free University of Bozen- } \\ & \text { bolzano }\end{array}$

\section{T. Elias} (2010)

\section{E. Pearson, V.} Gkatzidou, and S. Green (2011)

\section{Universal Instructional Design Principles for Moodle}

Widgets to Support the Concept of an Adaptable Personal Learning Environment
Estudante sem especificação
Universal Design for Learning Universal Design of Instruction

\section{Estudantes do \\ Universal Design for Learning ensino superior Universal Design of Instruction}

Estudante sem Agile development especificação methodology to create Widgets

\section{Anido-Rifon, M.}

Fernandez-Iglesias,

C. Rivas-Costa, S.

Valladares-Rodriguez, and M. Gomez-

Carballa

(2013)

Providing a holistic educational environment for the whole family
Estudante sem
especificação

Adaptação de periféricos
B. Kouninef, G. Merad, and M. Djelti (2015)
The Use of QR Codes and Mobile Technology in the Blended Learning Approach
Estudante sem especificação
Mobile Learning

Tabela 2 - Caraterização dos artigos selecionados 
É de salientar que praticamente na totalidade dos 9 artigos analisados foi observada a importância dos padrões de acessibilidade, estabelecidos pelo $\mathrm{W}_{3} \mathrm{C}$, na implementação dos AVA.

\section{A acessibilidade em ambientes virtuais de aprendizagem}

Na educação não-formal, que decorre ao longo da vida, o processo ensino/aprendizagem desenvolve-se em diversos contextos e situações (Gohn, 2006), Neste contexto a acessibilidade dos AVA, para pessoas com diferentes perfis e necessidades assume uma preocupação relevante.

$\mathrm{Na}$ Tabela 2 resumem-se as principais caraterísticas dos artigos selecionados para esta revisão sistemática da literatura

Amado-Salvatierra et al. (Hector R. Amado-Salvatierra, Hernández, \& Hilera, 2012) (H R Amado-Salvatierra, Hernández, \& Hilera, 2014) (H R Amado-Salvatierra \& Rizzardini, 2014) descrevem um estudo de caso onde propõem a implementação de várias diretrizes e recomendações para o desenvolvimento de metodologias na produção de conteúdos multimédia/interativos, numa arquitetura de serviços web aberta, que visa apoiar a interação e garantir os principais padrões de acessibilidade para estudantes do ensino superior. O projeto ESVI-AL (Educación Superior Virtual Inclusiva - América Latina) e o projeto EU4ALL (European Unified Approach for Accessible Lifelong Learning) baseiam-se na norma UNE 66181, que oferece um conjunto de métodos e padrões genéricos de acessibilidade. Estes projetos implementaram e validaram um conjunto de requisitos de acessibilidade no contexto da educação em ambientes virtuais, reconhecidas como normas de boas práticas e padrões de qualidade.

McAndrew \& Cooper n.d (McAndrew \& Cooper, sem data) propõem o conceito de Universal Design for Learning (UDL). O UDL corresponde a um conjunto de princípios e estratégias que procuram reduzir as barreiras no ensino/aprendizagem, independentemente das necessidades físicas e cognitivas dos educandos universitários. Nesse contexto, o professor deve desenvolver planos de trabalho que possam proporcionar múltiplos meios: i) de Apresentação; ii) de Ação e Expressão; iii) de Autoenvolvimento (Engagement), enriquecendo as estratégias de ensino e promovendo atividades contextualizadas na prática pedagógica.

Para Ravanelli \& Serina 2014 (Ravanelli \& Serina, 2014)the contribution of technology, especially web 2.0, has transformed the concept of distance learning into that of e-learning and online learning. These are based on the use of CSCL (Computer Supported Collaborative Learning e Elias 2010 (Elias, 2010) a acessibilidade nos Learning Management System (LMS) passa pelo princípio do UDL e do Universal Design of Instruction (UDI). Este modelo permite construir módulos educativos ou planificar aulas de forma a aumentar a capacidade de aprendizagem, tornando-os mais eficazes e adequados a qualquer aluno, em diferentes contextos. De uma forma muito breve, o UDI enuncia oito princípios fundamentais de acessibilidade:

- Uso equitativo,

- Flexibilidade,

- Simplicidade, 
- Ser intuitivo,

- Ter informação percetível,

- Ter tolerância ao erro,

- Requerer um esforço físico e/ou técnico atingível,

- Ser acessível a uma comunidade ampla de alunos.

Deste modo, todos poderão interagir com os AVA, independentemente das suas limitações.

Dos artigos incluídos, apenas Pearson et al. 2011 (Pearson, Gkatzidou, \& Green, 2011) aborda as Widgets como uma ferramenta de apoio aos AVA vocacionados para o ensino/ aprendizagem de alunos com necessidades especiais.

A possibilidade de o aluno controlar o ambiente, transformando-o num Adaptable Personal Learning Environment (APLE) com recurso às widgets, e assim poder decidir que ferramentas e/ou serviços usar e agregar no/ao seu espaço pessoal é uma mais-valia para o processo de aprendizagem. Uma solução tecnológica suportada por Widgets, parametrizando o seu Personal Learning Environment (PLE), quer ao nível da definição de uma estrutura coerente de menus e submenus, quer ao nível das configurações de acessibilidade, está subjacente no projeto Widgets for Inclusive Distributed Environments (WIDE). O projeto visa desenvolver recursos que ampliem as funcionalidades dos AVA de forma a responderem às necessidades específicas de aprendizagem dos alunos, adotando assim um conjunto de novas metodologias na sua construção (Pearson et al., 2011).

Para L. Anido-Rifon et al. (L. Anido-Rifon, Fernandez-Iglesias, Rivas-Costa, ValladaresRodriguez, \& Gomez-Carballa, 2013), o objetivo é criar um AVA acessível através de diferentes periféricos para facilitar e/ou promover o seu acesso a qualquer aluno, independentemente da sua situação de dependência pessoal. É importante compreender o carácter circunstanciado deste exemplo de boas práticas que constituirá, em função dos requisitos associados aos mesmos, o acesso por parte de todas as pessoas a um AVA. Este conceito técnico permite uma abordagem holística destes ambientes educacionais.

Mais centrado na inovação ao nível da acessibilidade e comunicação dos LMS, Kouninef et al. (Kouninef, Merad, \& Djelti, 2015) descrevem os novos horizontes para o processo de ensino e aprendizagem utilizando os Quick Response Code (QR). O uso destes códigos poderá dar um novo enfoque à acessibilidade e à motivação dos alunos, introduzindo uma nova dinâmica nos AVA. O uso dos podcasts é um recurso pedagógico que possibilita um nível de interatividade elevado com os AVA. Os códigos QR poderão auxiliar e apoiar para todos os que têm dificuldades de compreensão dos processos de ensino/aprendizagem, independentemente das restrições de tempo e de lugar.

Todos os artigos citados abordam de uma forma muito explicita e sucinta alguns métodos necessários para a construção de AVA e oferecem um conjunto de procedimentos e padrões genéricos de acessibilidade, contribuindo para uma utilização mais ampla e abrangente destes ambientes. Amado-Salvatierra et al (2012, 2014 \& 2014) utilizam o modelo ESVIAL baseando numa série de métodos e normas de acessibilidade. McAndrew P, Ravanelli F, Elias T et al (2010, 2012 \& 2014) aplicaram o Universal Design for Learning e Universal Instructional Design na produção de AVA e utilizando princípios, estratégias e métodos educativos de forma a diminuir os obstáculos cognitivos na utilização dos AVA, facilitando a 
interação aluno-AVA. Pearson E (2011) utilizou um método Agile para desenvolver Widgets destinadas a serem utilizadas em AVA de forma a torná-los acessíveis. AnidoRifon L (2013) preocupou-se com a adaptação e criação de periféricos. E Kouninef L (2015) optou pelo uso de códigos para facilitar a acessibilidade e a motivação dos alunos.

\section{Discussão}

Apesar da inegável relevância do tema da acessibilidade digital, a necessidade de compilar de forma sistemática um conjunto de métodos é um dos fatores importantes e primordiais dos AVA.

A criação de AVA acessíveis é uma tarefa complexa. Envolve diversas áreas do saber:

- O Conhecimento Pedagógico do Conteúdo (PCK): a capacidade de ensinar um determinado conteúdo;

- O Conhecimento Tecnológico do Conteúdo (TCK): saber selecionar os recursos tecnológicos mais adequados para comunicar um determinado conteúdo;

- O Conhecimento Tecnológico-Pedagógico (TPK): saber usar esses recursos tecnológicos no processo de ensino-aprendizagem.

De acordo com Mishra e Koehler (Mishra \& Koehler, 2006) as relações entre o conteúdo, pedagogia e tecnologia são complexas. Neste contexto e baseados na formulação de Shulman (Shulman, 1986) do conhecimento pedagógico do conteúdo (PCK), criaram um modelo, denominada Technological Pedagogical Content Knowledge (TPACK), Figura 2.

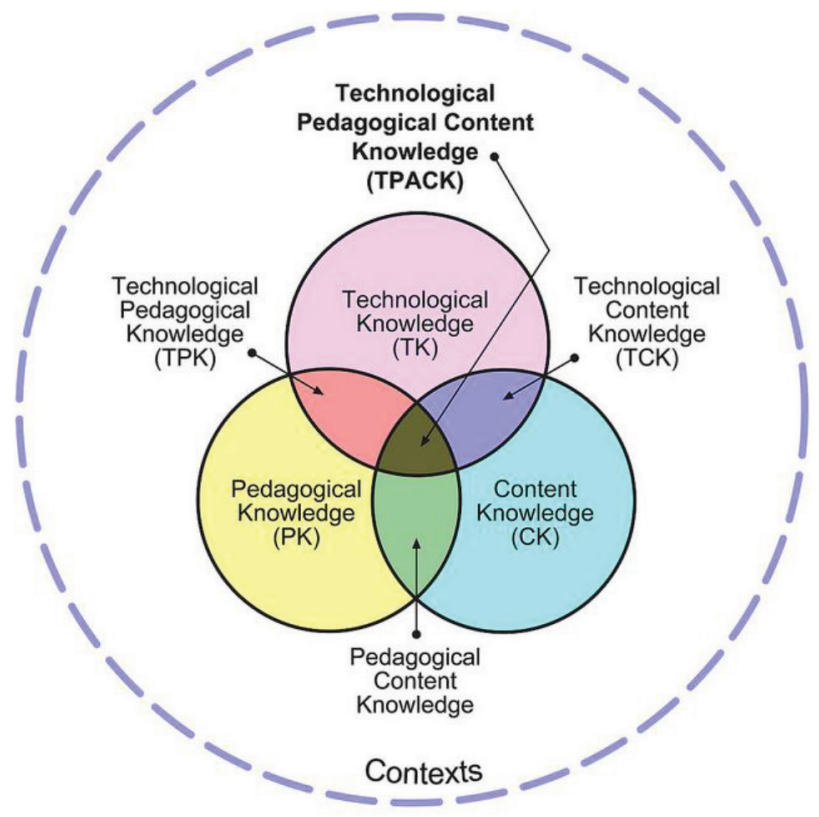

Figura 2 - Modelo TPACK

Fonte: http://tpack.org 
A interseção destes três domínios do conhecimento na criação dos AVA - TPACK -é uma das formas de potencializar a sua acessibilidade. Analisar questões que não fazem parte deste modelo é essencial para os AVA. Neste contexto, as Web Content Accessibility Guidelines (WCAG 2.0) abrangem um vasto conjunto de recomendações com o objetivo de tornar os conteúdos digitais mais acessíveis.

A solução para estas questões, aparentemente simples, impõe um complexo processo metodológico que passa pela aquisição da informação - realização de entrevistas, observação etnográfica e criação de grupos de discussão e pelo desenvolvimento de modelos baseados em personas, como objetivo fundamental de compreender e estudar os utilizadores, o seu contexto, avaliar e validar AVA - Contextual Design (Karen, Holtzblatt and R. Beyer, 1998).

Nos artigos selecionados - Tabela 2, foram diversos os métodos descritos/utilizados pelos autores para tomar os AVA mais acessíveis. O modelo TPACK e as diretrizes de acessibilidade da WCAG 2.0 estiveram na base da maioria das metodologias descritas.

Se na metodologia utilizada as diferenças entre os trabalhos foram relativamente pequenas, já na forma de estudar os utilizadores registámos uma grande diversidade, nem sempre os procedimentos do Contextual Design foram respeitados.

A combinação de um conjunto de boas práticas estão plasmadas nos projetos ESVIAL e EU4ALL (Hector R. Amado-Salvatierra et al., 2012) (H R Amado-Salvatierra et al., 2014) (H R Amado-Salvatierra \& Rizzardini, 2014), que desenvolveram um quadro geral aberto, baseado em padrões, para apoiar os serviços personalizados de ensino/ aprendizagem no contexto do ensino superior e na aprendizagem ao longo da vida.

No que concerne às práticas pedagógicas, o UDL (McAndrew \& Cooper, sem data) e UID (Ravanelli \& Serina, 2014)the contribution of technology, especially web 2.0, has transformed the concept of distance learning into that of e-learning and online learning. These are based on the use of CSCL (Computer Supported Collaborative Learning e (Elias, 2010) permitem identificar e fundamentar a conformidade das ações na planificação e organização dos AVA com a identificação de metodologias de ensino facilitadoras da inclusão, da participação e do ensino/aprendizagem para todos (National Center On Universal Design For Learning, 2013), embora

A possibilidade de incorporar e parametrizar ferramentas de acessibilidade (Widgets), desenvolvidas de acordo com as linhas orientadoras da "Metodologia Ágil" (Pearson et al., 2011), permite o desenvolvimento de Widgets totalmente inovadoras e a sua integração nos AVA.

Outro aspeto a salientar é a necessidade da utilização de periféricos alternativos ou adaptados, proporcionando um controlo simplificado e ajustável a cada aluno com necessidades específicas para acesso aos recursos oferecidos nos AVA (L. Anido-Rifon et al., 2013).

O uso dos códigos QR poderá quebrar um conjunto de barreiras físicas e cognitivas associadas ao ensino/aprendizagem em AVA, oferecendo uma nova oportunidade a todos os alunos. Este recurso pedagógico enquadra-se nos princípios do Mobile Learning (ML) e consegue, de uma forma ubíqua, cruzar informações entre diferentes AVA, permitindo um novo e dinâmico modelo de interação Homem-máquina (Kouninef et al., 2015) . 


\section{Considerações finais}

Este artigo teve como objetivo principal apresentar e sintetizar o conhecimento disponível, envolvendo alguns dos métodos existentes para a criação de AVA mais acessíveis. Desta forma seria possível aperfeiçoar e responder melhor às necessidades educacionais dos utilizadores dos AVA.

Procurou-se dar um pequeno contributo para esta temática com o intuito de tornar o ensino/aprendizagem mais eficaz, eficiente e aprazível, proporcionando novas oportunidades de aprendizagem e reforçando a relação aluno-professor.

Com efeito, e independentemente do conceito de acessibilidade e de AVA, é importante reter que a combinação destes métodos permite uma melhoria na acessibilidade dos ambientes de aprendizagem. Estes processos complexos que implicam interação Homem-máquina exigem a integração de recursos tecnológicos acessíveis com práticas pedagógicas.

Compreender e estudar os utilizadores, o seu contexto (Karen, Holtzblatt and R. Beyer, 1998) e simplificar a complexidade da informação, tornando-a percetível a todos os alunos (Wurman, 2001) (Rosenfeld, Morville, \& Arango, 2006), são objetivos fundamentais dos trabalhos de investigação e criação destes novos ambientes de aprendizagem.

Existem alguns métodos para a construção de AVA acessíveis que não foram identificados em nenhum dos artigos consultados. Este facto deve-se à circunstância de a pesquisa ter sido efetuada com uma conjugação de palavras-chaves e operadores que tinha como objetivo centrar a questão na acessibilidade e nos AVA.

Outro dos aspetos não abordados em muitos dos artigos e de grande relevância para avaliar o nível de acessibilidade dos AVA constitui a definição de critérios de avaliação, a metodologia dos testes e as ferramentas utilizadas para tal.

\section{Referências bibliográficas}

Amado-Salvatierra, H. R., Hernández, R., \& Hilera, J. R. (2012). Implementation of Accessibility Standards in the Process of Course Design in Virtual Learning Environments. Procedia Computer Science, 14, 363-370. https://doi.org/10.1016/j. procs.2012.10.042

Amado-Salvatierra, H. R., Hernández, R., \& Hilera, J. R. (2014). Teaching and promoting web accessibility in virtual learning environments: A staff training experience in Latin-America. In 2014 IEEE Frontiers in Education Conference (FIE) Proceedings. Madrid, Spain: IEEE. https://doi.org/10.1109/FIE.2014.7044392

Amado-Salvatierra, H. R., \& Rizzardini, R. H. (2014). Towards a methodology to inclusive curriculum design: An experience presented within an accessible virtual learning environment. In 2014 IEEE Frontiers in Education Conference (FIE) Proceedings. Madrid, Spain: IEEE. https://doi.org/10.1109/FIE.2014.7044468 
Anido-Rifon, L. Fernandez-Iglesias, M., Rivas-Costa, C., Valladares-Rodriguez, S., \& Gomez-Carballa, M. (2013). Providing a holistic educational environment for the whole family. 2013 IEEE Frontiers in Education Conference (FIE), Frontiers in Education Conference, 2013 IEEE. IEEE. https://doi.org/10.1109/ FIE.2013.6685029

Center, T. R. \& D. (2013). About the Trace Center - Trace Center. Obtido 17 de Abril de 2016, de http://trace.wisc.edu/about/

Dillenbourg, P., Schneider, D., \& Synteta, P. (2002). Virtual Learning Environments. In Proceedings of 3 rd Hellenic Conference" Information \& Communication Technologies in Education" (p. 3-18). Rhodes, Greece.

Elias, T. (2010). Universal Instructional Design Principles for Moodle. International Review of Research in Open and Distance Learning, 11(2), 110-124. Obtido de http://widgets.ebscohost.com/prod/customerspecific/nsooo290/authentication/ index.php?url=http\%3A\%2F\%2Fsearch.ebscohost.com\%2Flogin.aspx\%3Fdirect\% 3Dtrue\%26AuthType\%3Dip\%2Ccookie\%2Cshib\%2Cuid\%26db\%3Deric\%26AN\%3 DEJ895752\%26lang\%3Dpt-br\%26site\%3Deds-live\%26sc

Gohn, M. da G. (2006). Educação não-formal, participação da sociedade civil e estruturas colegiadas nas escolas. Revista Ensaio-Avaliação e Políticas Públicas em Educação, 14(50), 11-25.

Holtzblat, K., \& Beyer, H.R. (1998). Contextual Design. Obtido 10 de Setembro de 2017, de https://www.interaction-design.org/literature/book/the-encyclopedia-ofhuman-computer-interaction-2nd-ed/contextual-design

Keller, C. (2005). Virtual learning environments: three implementation perspectives. Learning, Media and Technology, 30(3), 299-311. https://doi. org/10.1080/17439880500250527

Kouninef, B., Merad, G., \& Djelti, M. (2015). The Use of QR Codes and Mobile Technology in the Blended Learning Approach. In Proceedings of 2015 Fifth International Conference on e-Learning (econf), e-Learning (econf), 2015 Fifth International Conference on, econf. Manama, Bahrain: IEEE. https://doi.org/10.1109/ ECONF.2015.90

Liberati, A., Altman, D. G., Tetzlaff, J., Mulrow, C., Gøtzsche, P. C., Ioannidis, J. P. A., ... \& Moher, D. (2009). The PRISMA statement for reporting systematic reviews and meta-analyses of studies that evaluate health care interventions: explanation and elaboration. Journal of clinical epidemiology, 62(10), e1-34. https://doi. org/10.1016/j.jclinepi.2009.06.006

McAndrew, P., \& Cooper, R. F. M. (2012). Adapting online learning resources for all: planning for professionalismin accessibility. Researchin LearningTechnology, 20(0), 1-17. Obtido de http://search.ebscohost.com/login.aspx?direct=true\&site=eds-liv e\&db=edsdoj\&AN=edsdoj.9dafb40cd674fc497da1016671b7ac7 
Mishra, P., \& Koehler, M. J. (2006). Technological Pedagogical Content Knowledge: A Framework for Teacher Knowledge. Teachers College Record. Vol 108(6), 1017-1054.

Moher, D., Liberati, A., Tetzlaff, J., Altman, D. G., \& Grp, P. (2009). Preferred Reporting Items for Systematic Reviews and Meta-Analyses: The PRISMA Statement (Reprinted from Annals of Internal Medicine). Physical Therapy, 89(9), 873-880. https://doi.org/10.1371/journal.pmed.1000097

National Center On Universal Design For Learning. (2013). The Concept of UDL. Obtido 31 de Maio de 2017, de http://www.udlcenter.org/aboutudl/whatisudl/ conceptofudl

Pearson, E., Gkatzidou, V., \& Green, S. (2011). Widgets to Support the Concept of an Adaptable Personal Learning Environment. In Proceedings of 2011 IEEE 11th International Conference on Advanced Learning Technologies. https://doi. org/10.1109/ICALT.2011.178

Ravanelli, F., \& Serina, I. (2014). Didactic and Pedagogical View of E-learning Activities Free University of Bozen-bolzano. Procedia - Social and Behavioral Sciences, 116, 1774-1784. https://doi.org/10.1016/j.sbspro.2014.01.471

Rocha, Á. (2012). Framework for a global quality evaluation of a website. Online Information Review, 36(3), 374-382. https://doi.org/10.1108/14684521211241404

Rodrigues, S., Rocha, Á., \& Abreu, A. (2017). The use of moodle in higher education evolution of teacher's practices over time. In Information Systems and Technologies (CISTI), 2017 12th Iberian Conference on (pp. 1-4). IEEE. https://doi.org/ 10.23919/ CISTI.2017.7975702

Rosenfeld, L., Morville, P., \& Arango, J. (2006). Information Architecture for the World Wide Web. Boston, MA: O’Reilly Media.

Shulman, L. S. (1986). Those Who Understand: Knowledge Growth in Teaching. EducationalResearcher,15(2),4-14.https://doi.org/10.3102/0013189X015002004

W3C. (2005). Introdução à Acessibilidade Web. Obtido 27 de Abril de 2016, de https:// www.netfolio.pt/w3c/WAI_intro_acessibilidade

Wurman, R. S. (2001). Information Anxiety 2. Plymouth, MI: Hayden Books/ Que Publishing. 